\section{Looking inside quantum dots}

\section{Eaves}

MINIATURIZATION in microelectronics has reached a point where researchers are trying to create quantum dots, more fancifully dubbed artificial atoms, embedded in semiconductor structures. The energy levels of electrons in these dots, as in real atoms, are discrete not continuous, but can be varied at will by the experimenter. R. C. Ashoori et al. now show (Phys. Rev. Lett. 68, 30883091 ; 1992) that it is possible to measure the spectrum of a quantum dot by recording the small current that flows as a single electron enters or leaves it.

The techniques that make this research possible are the techniques that have given us key components in two of the most popular products in the consumer electronics market - compactdisk players and satellite-television receiver dishes. The essential feature of the components (quantum-well lasers and high-electron-mobility transistors) is the two-dimensional nature of their electronic states. Confinement provided by planar potential-energy barriers in the semiconductor structure constrains electrons to move freely in two dimensions only. In the third direction, perpendicular to the barriers, the motion is fully quantized - one-half of a de Broglie wavelength fits into the narrow quantum well (perhaps $100 \AA$ across) separating the two barriers. This lowering of the dimensionality of the electron motion
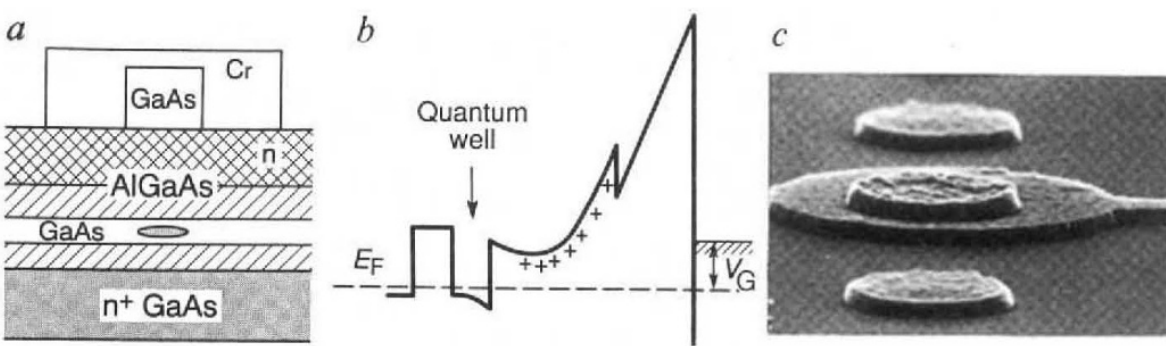

FIG. 1 a, A schematic cross-section through Ashoori et al.'s heterostructure, sandwiched by a chromium and a high-conductivity ( $\mathrm{n}^{+} \mathrm{GaAs}$ ) semiconductor electrode. The quantum dot (shaded ellipse) is confined in the GaAs quantum well. $b$, Potential energy diagram showing the potential well provided by the sandwiched GaAs layer, and the Fermi energy $\left(E_{\mathrm{F}}\right) . C_{1}$ Micrograph (2.5um across) of the device, showing the top chromium gate.

gives rise, in part, to the components' special properties.

Confinement allows physicists and electronic engineers to reduce the dimensionality further, to create the quantum dot in which the electrons' motion is restricted and quantized along all three directions. In this case the energy states of the electrons in the semiconductor should occupy discrete levels rather than the continuous energy bands which are characteristic of bulk solids. In other words, quantum dots should have atom-like energy levels, but

with the prospect that the level separation can be modified in a controllable way.

The quantum dot of Ashoori et al. is based on a two-dimensionally confined electron gas, formed in a thin $(15 \mathrm{~nm})$ quantum-well layer of gallium arsenide (GaAs) sandwiched between two layers of aluminium gallium arsenide (AlGaAs). The additional confinement is provided by applying a voltage to a chromium-metal gate electrode deposited on the surface of the semiconductor heterostructure. When this is biased negative, the electronic motion in the quantum well is restricted to a diskshaped quantum dot with a diameter of less than $1 \mu \mathrm{m}$ (Fig. 1). As the gate voltage is altered, electrons can enter the dot from a high-conductivity $\mathrm{n}^{+} \mathrm{GaAs}$ layer by quantum-mechanical tunnelling through the lower barrier, which is only $85 \AA$ across.

The tunnelling occurs only when the characteristic 'Fermi' energy of the electrons in the lower GaAs layer equals one of the distinct energy levels of the quantum dot. This allows Ashoori et al. to measure the 'spectrum' of their artificial atom. They start their experiment with the quantum dot empty. By steadily decreasing the gate voltage, $V_{\mathrm{G}}$, they can add electrons one-by-one to the quantum dot. The particular voltages, $V_{\mathrm{G}}$, at which this occurs correspond to observed. Each of the smaller $p$ (such as those at gate voltages around $-600 \mathrm{mV}$ ) arises from a single electron tunnelling into one of the low-energy discrete energy levels of the quantum

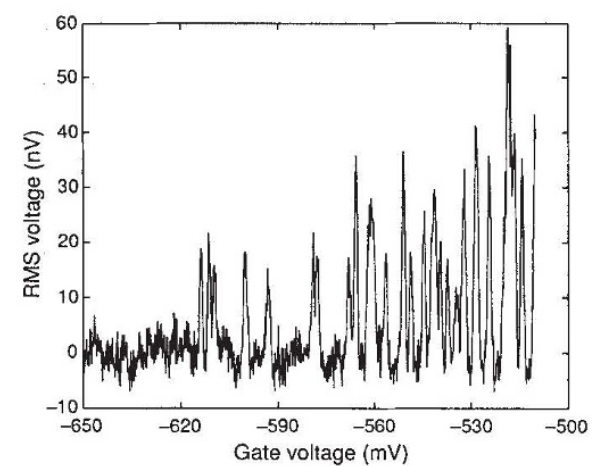

FIG. 2 The root-mean-square signal as a function of gate bias using $0.33-\mathrm{mV}$ a.c. excitation. A linear background has been subtracted.

well. The trace therefore represents a direct energy-level spectrum of the quantum dot. The spacing of the peaks in Fig. 2 does not correspond exactly to the spacing of the energy levels, owing to an 'electrostatic leverage' effect.

But the characteristic level spacing is a few millielectronvolts. This is too large a value to arise from the quantum confinement provided by the electrostatic potential of the gate itself.

Instead, it is thought that potentialenergy fluctuations in the quantum well create 'puddles' which further localize the electrons. This interpretation is supported by investigating the variation of the peak position $\left(V_{\mathrm{G}}\right)$ when a large magnetic field is applied perpendicular to the plane of the two-dimensional electron gas. The effect of the field is to provide even stronger confinement on the energy puddles. By looking at how the spectrum varies with applied field, the authors identify two scales for the potential puddles, both attributable, they suggest, to silicon atoms used to dope the GaAs electrode. Weak, shallow puddles, responsible for confinement energies of $0.3-2.5 \mathrm{meV}$, are probably the result of variations in silicon concentration in or near the GaAs electrode.

Stronger confinement, indicated by a weak dependence on applied field, is probably due to silicon atoms that migrated from the electrode into the well as the device was fabricated. Indeed, my colleagues have found that individual impurity atoms in quantum dots can completely dominate many of the dot's characteristics (M. W. Dellow et al. Phys. Rev. Lett. 68, 1754-1759; 1992; see S. Washburn's News and Views, Nature 357, 199-200; 1992; and also P. Guéret et al. Phys. Rev. Lett. 68, 18961899; 1992 for a contrary view). The ultimate, unadulterated artificial atom is yet to be created.
L. Eaves is in the Department of Physics, University of Nottingham, Nottingham NG7 2RD, UK. 